\title{
Efficacy, Safety, and Long-Term Follow-Up Results of EUS-Guided Transmural Drainage for Pancreatic Pseudocyst
}

\author{
Shin Kato, Akio Katanuma, Hiroyuki Maguchi, Kuniyuki Takahashi, \\ Manabu Osanai, Kei Yane, Toshifumi Kim, Maki Kaneko, Ryo Takaki, \\ Kazuyuki Matsumoto, Tomoaki Matsumori, Katsushige Gon, and Akiko Tomonari
}

Center for Gastroenterology, Teine-Keijinkai Hospital, Sapporo 006-8555, Japan

Correspondence should be addressed to Shin Kato; shinchan1231@gmail.com

Received 9 September 2012; Revised 13 February 2013; Accepted 13 February 2013

Academic Editor: Charles Melbern Wilcox

Copyright (C) 2013 Shin Kato et al. This is an open access article distributed under the Creative Commons Attribution License, which permits unrestricted use, distribution, and reproduction in any medium, provided the original work is properly cited.

\begin{abstract}
Background and Aim. EUS-guided transmural drainage (EUS-GTD) is now considered a minimally invasive and effective alternative to surgery for drainage of symptomatic pancreatic pseudocysts. However, the technique is rather difficult, and sometimes serious complications occur to patients undergoing this procedure. We retrospectively evaluated efficacy, safety, and long-term follow-up results of EUS-GTD for pancreatic pseudocyst. Methods. Sixty-seven patients with pancreatic pseudocyst who underwent EUSGTD from April 2000 to March 2011 were enrolled. We retrospectively evaluated (1) technical success, (2) clinical success, (3) adverse event of procedure, and (4) long-term follow-up results. Results. Total technical success rate was $88 \%$. Ninety-one percent of external drainage, $79 \%$ of internal drainage, and $66 \%$ of puncture and aspiration only achieved clinical success. There was only one case with an adverse event, perforation (1.5\%). The case required emergency operation. Total recurrence rate was $23.9 \%$. Median follow-up period was 33.9 months. The recurrence rates in the cases of stent remaining, spontaneously dislodged, removed on schedule, external tube removal, and aspiration only were $10.0 \%, 12.5 \%, 42.9 \%, 50 \%$, and $0 \%$, respectively. Conclusion. EUS-GTD is a relatively safe and effective therapeutic method. However, further analysis should be done by larger series to determine the method of EUS-GTD for pancreatic pseudocyst.
\end{abstract}

\section{Introduction}

Endoscopic ultrasonography guided transmural drainage (EUS-GTD) is now a widely reported and established procedure for drainage of symptomatic pancreatic pseudocyst. The technical success rate of this procedure has been reported to be more than $90 \%$ on the other hand, complication rate is less than $5 \%$ [1-5]. Severe adverse events such as bleeding or perforation sometimes occur, but in general, EUS-GTD has been recognized as a relatively safe and effective therapeutic method and is performed as a common procedure in many institutes. However, some factors including the long-term outcome, appropriate period of stenting, and timing of removing the stent have not been determined yet.

\section{Patients and Methods}

Patient characteristics are described in Table 1. Sixtyseven patients with pancreatic pseudocyst who underwent
EUS-GTD from April 2000 to March 2011 were enrolled. Indications of EUS-GTD at our center are as follows: symptomatic pancreatic cysts, infection or hemorrhage, rapid increase in size, and no communication with MPD. In this study, 3 cases after acute pancreatitis (type 1) had walled off necrosis. Abdominal computed tomography and transabdominal ultrasonography had been taken in all cases before EUS-GTD.

All procedures were performed using curved linear array EUS (GF UCT-240, GF UCT-260 Olympus Medical Systems, Tokyo, Japan). The used needles are EchoTip Ultra 19G (Cook Medical Inc., Winston Salem, NC, USA), GW, Visiglide 0.025 inch (Olympus Medical Systems), Jagwire 0.035 inch (Boston Scientific Japan, Tokyo, Japan), dilation catheter, Needlecut 3V (Olympus Medical Systems), and Soehendra biliary dilation catheter (Cook Medical Inc.). We used Pigtail nasobiliary catheter (6,7 Fr, Olympus Medical Systems. 7.5 Fr, Boston 
TABLE 1: Clinical characteristics of the patients.

\begin{tabular}{lc}
\hline Gender M : F & $50: 17$ \\
Median age, yr (range) & $56(31 \sim 85)$ \\
Median cyst size, diameter in mm (range) & $72(19-186)$ \\
Cyst location & 23 \\
$\quad$ Head & 44 \\
$\quad$ Body and tail & \\
Classification & 15 \\
Type I*: acute pancreatitis & 14 \\
Type II: acute exacerbation of chronic pancreatitis & 26 \\
Type III: retention cyst & 12 \\
Type IV: pancreatic fistula after pancreatic surgery & \\
\hline
\end{tabular}

*3 cases in type I had walled off necrosis.

Scientific Japan) for external drainage and 7 Fr double pigtail catheter (Olympus Medical Systems) for internal drainage.

We evaluated (1) technical success rate of initial drainage,

(2) Clinical Success rate, (3) adverse event of procedure, and

(4) long-term follow-up results after EUS-GTD.

\section{Technique of EUS-GTD}

All procedures were performed in the fluoroscopy unit under given intravenous sedative agent. Following steps were used to perform EUS-GTD.

The cystic lesion of pancreas was examined with ultrasonographically, and doppler assessment of gastric or duodenal wall was performed to confirm the absence blood vessels at the puncture site. Then, pancreatic pseudocyst was punctured with EchoTip 19G needle (Figure 1(a)) and the GW was passed through the needle into the lumen of the cyst. The position of GW was confirmed by ultrasonography and fluoroscopy (Figure 1(b)). After placing the GW, electrocautery was applied with NeedleCut3V, and dilation was achieved with a Soehendra biliary dilation catheter (Figure 1(c)). After dilatation, 2nd GW was inserted through Soehendra biliary dilation catheter, and plastic stent and drainage tube were placed under the guidance of GW. 7Fr double pigtail stent was used for internal drainage and $6 \mathrm{Fr}$ ENBD pigtail catheter for external drainage (Figures 1(d) and $1(\mathrm{e})$ ).

We mainly chose external drainage. However, in cases where patients were likely to remove the tube by themselves or there was no infected fluid in the cyst, we chose internal drainage. In some cases with pseudocyst with small size and no infected fluid, we performed only puncture and aspiration. From April 2010, we inserted both internal and external drainage tube at one procedure session.

\section{Results}

4.1. Technical Success. Total technical success rate was $88.1 \%$ (59/67) (Table 2). Technical success rates of transgastric approach and transduodenal approach were $88.3 \%$ and $75.0 \%$, respectively. Depending on the procedure, the success rates of external drainage, internal drainage, both internal
TABLE 2: Technical success rate of initial drainage.

\begin{tabular}{lcc}
\hline Access route & Stomach & $88.3 \%(53 / 59)$ \\
& Duodenum & $75 \%(6 / 8)$ \\
& External drainage & $83.7 \%(36 / 43)$ \\
Success rate of & Internal drainage & $95 \%(19 / 20)$ \\
procedure & Internal and external drainage & $100 \%(3 / 3)$ \\
& Puncture and aspiration only & $100 \%(1 / 1)$ \\
\hline Total $^{*}$ & & $88.1 \%(59 / 67)$ \\
\hline
\end{tabular}

*The rate was defined as the cases that the procedures accomplished as planned successfully. Failed 8 cases had puncture and aspiration later.

TABLE 3: Clinical success rate.

\begin{tabular}{lc}
\hline Initial drainage & Rate \\
\hline External drainage & $91.7 \%(33 / 36)$ \\
Internal drainage & $78.9 \%(15 / 19)$ \\
Internal and external drainage & $66.7 \%(2 / 3)$ \\
Puncture and aspiration only & $66.7 \%(6 / 9)^{*}$ \\
\hline Total & $83.4 \%(56 / 67)$ \\
\hline${ }^{*} 8$ cases (internal or external drainage failed) were included.
\end{tabular}

external drainage, and punctured and aspiration only were $83.7 \%, 95 \%, 100 \%$, and $100 \%$, respectively. Reasons for technical failure were difficulty in advancing the GW or its displacement in 2 cases, difficulty in achieving dilation in 4 cases, and difficulty with drainage tube placement in 2 cases. In these failed cases, we performed puncture and aspiration only.

4.2. Clinical Success. In our series, clinical success was defined as cases in which initial EUS-GTD contributed to cyst shrinking over $10 \mathrm{~mm}$ or improvement of clinical symptoms. Table 3 shows that clinical success rate of external drainage was $91.7 \%(33 / 36)$, internal drainage was $78.9 \%$, both internal and external drainage was $66.7 \%(2 / 3)$, and puncture and aspiration only was also $66.7 \%$ (6/9). Total clinical success rate was $83.4 \%$ (56/67). In the failed 3 cases of external drainage, 1 case had additional multiple stenting which contributed to cyst shrinking, and 2 cases had operation. The failed 4 cases of internal drainage had added external tube drainage and all cases achieved cyst shrinking consequently. The failed 1 case of external and internal drainage had additional multiple stenting which was effective. In the failed 3 cases of puncture and aspiration only, each case had either operation, ESWL, or EPS (Table 4). $92.5 \%$ of the cases $(62 / 67)$ achieved improvement by EUS-GTD.

4.3. Adverse Event of Procedure. The rate of adverse event was $1.5 \%(1 / 67)$. There was one case with perforation in our series. The perforation case had a type III pancreatic cyst (Figure 2(a)). Perforation occurred during balloon dilatation for multiple stents placement. After balloon dilatation, we recognized leakage of contrast medium and advancing of the GW into the abdominal cavity (Figures 2(b) and 2(c)). This case required surgical operation (Figure 2(d)). 


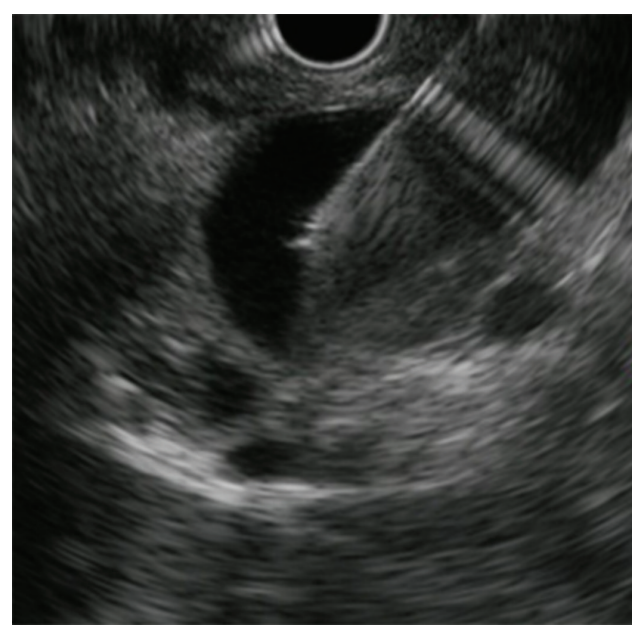

(a)

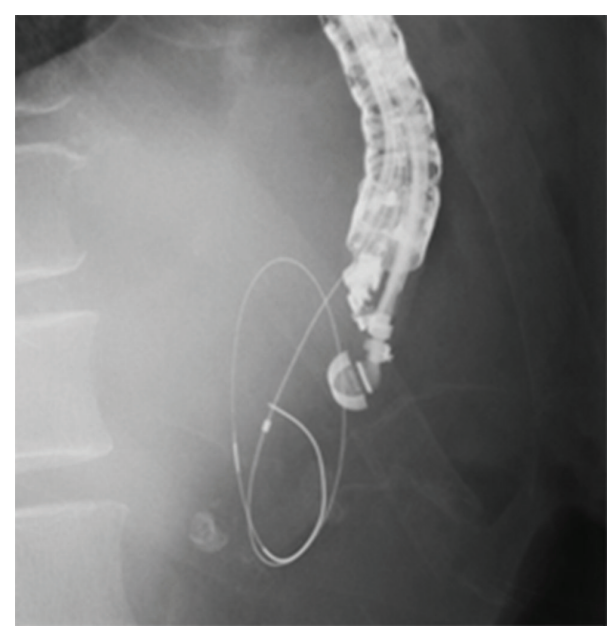

(b)

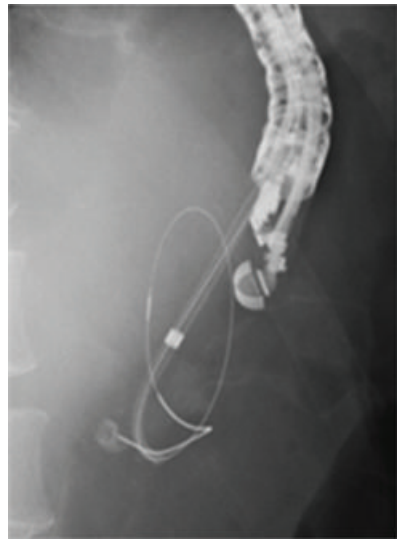

(c)

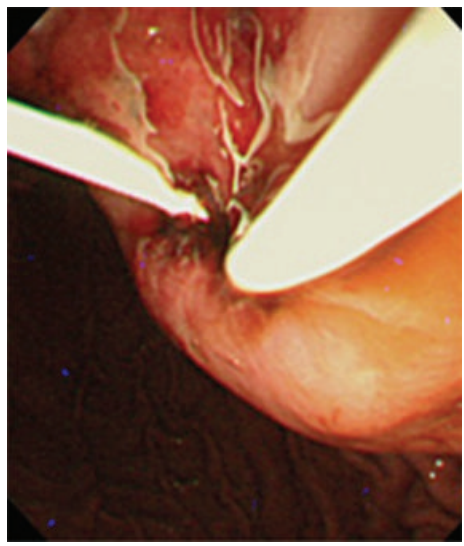

(d)

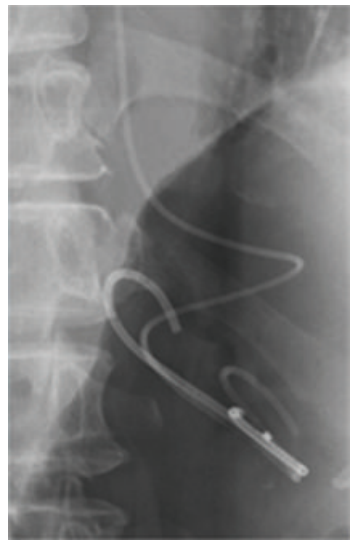

(e)

FIGURE 1: (a) Pancreatic pseudocyst was punctured with EchoTip $19 \mathrm{G}$ needle. (b) Fluoroscopy image showed that the guide wire was passed through the needle into the lumen of cyst. (c) After electrocautery, Soehendra dilation catheter was inserted into the lumen for dilatation. (d) and (e) 7 Fr double pigtail stent and 6 Fr ENBD pigtail catheter were placed.

TABLE 4: Management of failed cases in initial drainage.

\begin{tabular}{lccc}
\hline Initial drainage & $\begin{array}{c}\text { Failed } \\
\text { cases }\end{array}$ & Additional therapy & \\
\hline $\begin{array}{l}\text { External drainage } \\
(n=36)\end{array}$ & 3 & $\begin{array}{c}\text { Multiple stents } \\
\text { Repuncture* } \rightarrow \text { operation } \\
\text { Operation }\end{array}$ & 1 \\
& & Add external drainage & 1 \\
$\begin{array}{l}\text { Internal drainage } \\
(n=19)\end{array}$ & 4 & Multiple stents & 1 \\
$\begin{array}{l}\text { External and } \\
\text { internal drainage }\end{array}$ & 1 & ESWL & 1 \\
$(n=3)$ & & EPS & 1 \\
$\begin{array}{l}\text { Puncture and } \\
\text { aspiration only }(n=9)\end{array}$ & 3 & Operation & 1 \\
\end{tabular}

ESWL: extracorporeal shock wave lithotripsy. EPS: endoscopic pancreatic stent. ${ }^{*}$ CT guided-cyst drainage. ${ }^{* *}$ Operation for an adverse event caused by EUS-CD procedure.

4.4. Long-Term Follow-Up Results (Table 5). In the 46 cases which finally achieved clinical improvement by EUS-GTD and were observed for more than 1 year, overall recurrence rate was $23.9 \%$ (11/46). Median follow-up period of the above cases was 33.9 months. Final stent conditions were as follows: 40 cases had internal drainage (ID), 4 cases had external tube removal (stent free), and 2 cases had only puncture and aspiration. Stent was still remaining in 10 cases of ID and 1 of them had recurrences (10\%). In 16 cases of ID, stent was dislodged spontaneously, and 2 of them had recurrences (12.5\%). In 14 cases of ID, stents were removed on schedule and 6 of them had recurrence (42.9\%). 2 cases of aspiration only and external drainage tube removal had recurrences (20\%).

We also analyzed the factors of recurrence by comparing recurrence group $(n=11)$ and nonrecurrence group $(n=$ 35) (Table 6, Fischers test, $P$ value $<.05$ was considered statistically significant); however, no factors were significant to determining recurrences.

\section{Discussion}

The technical success rate of EUS-GTD has been reported to be more than $90 \%$ in several studies [1-5]. In our series, the 


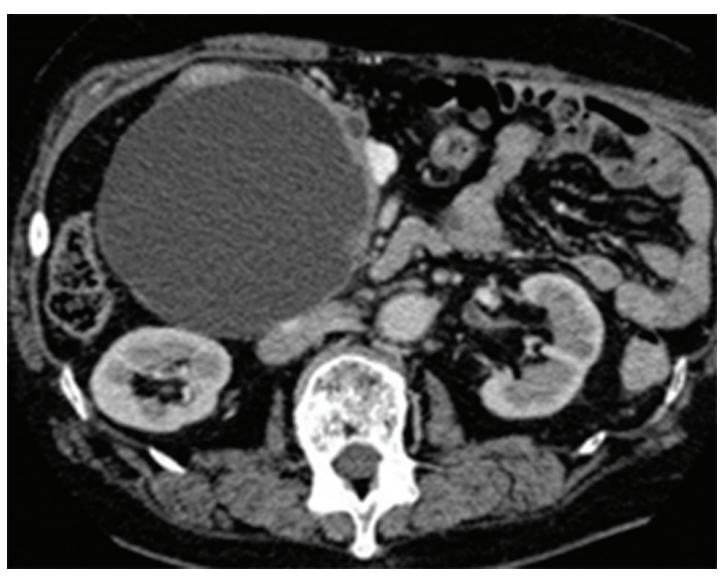

(a)

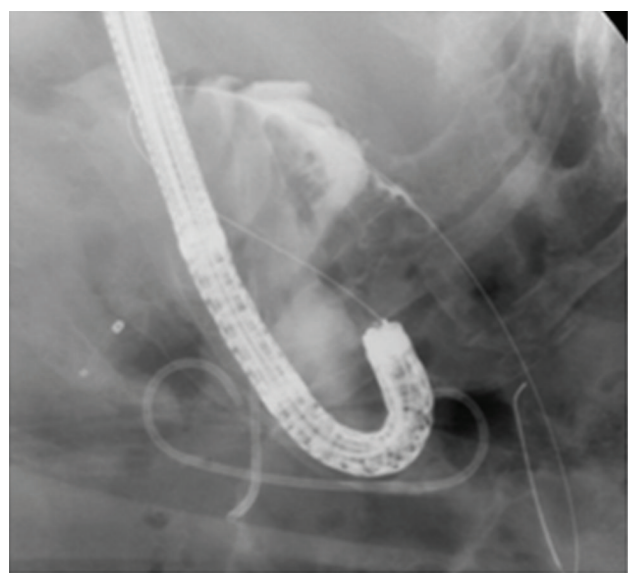

(c)

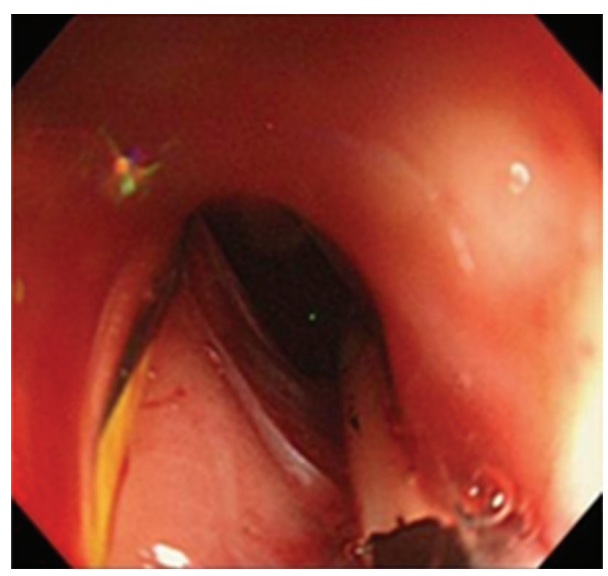

(b)

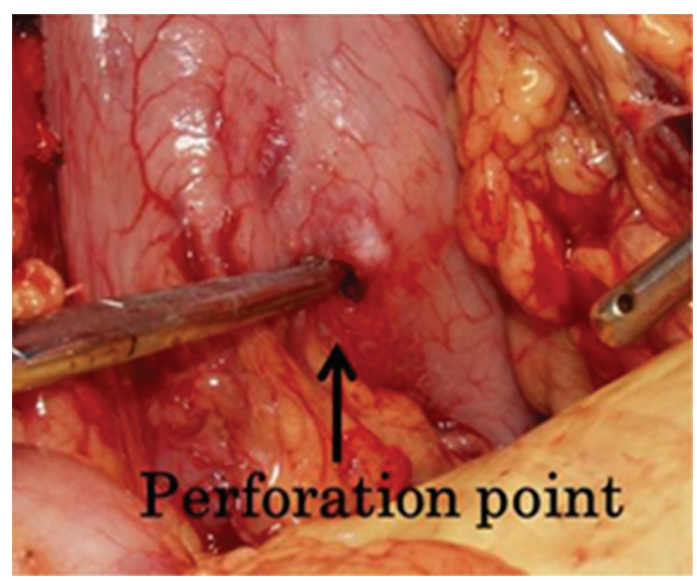

(d)

FIgURE 2: (a) Type III pancreatic pseudocyst. (b) The edge of omentum was observed through the hole dilated by balloon. (c) Leakage of contrast medium and advancing of the guide wire into the abdominal cavity were observed. (d) Perforation point was clearly confirmed by operation.

TABLE 5: Recurrence rate and stent condition.

\begin{tabular}{lccc}
\hline \multicolumn{2}{c}{ Recurrence rate $23.9 \%(11 / 46)$} & $n=46$ \\
Final drainage & Stent condition* & & Recurrence \\
\hline $\begin{array}{l}\text { Internal drainage } \\
(n=40)\end{array}$ & Still remaining & 10 & $1(10 \%)$ \\
$\quad \begin{array}{l}\text { Single stent } \\
(n=34)\end{array}$ & $\begin{array}{c}\text { Spontaneous } \\
\text { dislodged }\end{array}$ & 16 & $2(12.5 \%)$ \\
$\quad \begin{array}{l}\text { Multiple stent } \\
(n=6)\end{array}$ & Scheduled removal & 14 & $6(42.9 \%)$ \\
$\begin{array}{l}\text { Puncture and } \\
\text { aspiration only } \\
(n=2)\end{array}$ & Stent-free & 2 & $0(0 \%)$ \\
$\begin{array}{l}\text { External tube } \\
\text { removal } \\
(n=4)\end{array}$ & Stent-free & 4 & $2(50 \%)$ \\
\hline
\end{tabular}

*The duration of internal drainage: 0.5 97.1 (median 20) months.

technical success rate was $88.1 \%$. The technical success rate of external drainage was slightly low $(83.7 \%)$, but the rates of internal drainage and both internal and external drainage were relatively high (95\% and 100\%, resp.). Clinical success rate was $83.4 \%$, and by adding extra EUS-GTD procedure, $92.5 \%$ of the cases achieved clinical improvement. Our result also supported that EUS-GTD is an effective procedure for pancreatic pseudocyst.

It is stated in previous studies that some cases with walled off necrosis after acute pancreatitis need not only tube drainage but also debridement. The success rate of EUS drainage for pancreatic abscess or necrosis was lower compared with clear fluid pancreatic pseudocyst [6]. One of the recent studies also reported that multiple transluminal gateway technique for EUS guided drainage was clearly effective for walled off necrosis of pancreas [7]. In our series, 3 cases had walled off necrosis; however, all improved by only conservative external drainage without debridement.

Severe adverse events such as perforation and bleeding sometimes occur in EUS-GTD. The rate of adverse event was reported to be less than 5\% [5]. Our series also had 1 case with perforation and that case needed operation. In our case, perforation occurred when balloon dilatation was performed. Balloon dilatation method has been thought to be a safer method compared to using electrocautery [8]. However, if the 
TABLE 6: Evaluation of factors for recurrence.

\begin{tabular}{lccc}
\hline & $\begin{array}{c}\text { Recurrence } \\
(11)\end{array}$ & $\begin{array}{c}\text { Non- } \\
\text { recurrence } \\
(35)\end{array}$ & $P$ value \\
\hline Patients characteristics & 9 & 29 & 0.7064 \\
Male & 4 & 15 & 0.9756 \\
Age $<50$ & 4 & 8 & 0.6197 \\
Age $\geq 70$ & 3 & 11 & 0.9089 \\
Location of cyst, Ph & 2 & 6 & 0.7064 \\
Diameter of cyst $\geq 10 \mathrm{~cm}$ & 5 & 14 & 0.7485 \\
Infection & & & \\
Procedure & 9 & 31 & 0.9466 \\
Internal tube drainage & 2 & 2 & 0.4685 \\
External tube removal & 0 & 2 & 0.9706 \\
Puncture, aspiration only & 1 & 9 & 0.4551 \\
Stent remaining & 2 & 1 & 0.2732 \\
Initial clinical failure & 1 & 5 & 0.9744 \\
Multiple stenting & & & \\
\hline
\end{tabular}

cyst wall of pancreas and gastric wall are not adhered to each other, the risk of perforation is clearly increased. Pancreatic cyst of our perforation case was type III, and this seemed to be the main cause of perforation. In our institute, first step drainage for type III cyst is endoscopic transpapillary drainage except in cases with severe clinical symptoms such as cyst infection or abdominal pain. In 14 cases out of 26 type III cysts, we performed transpapillary drainage (ENPD) first, but the procedure was not effective. In 3 cases, we failed to place ENPD and performed transmural drainage only. There were 9 cases in which transmural drainage was directly performed due to severe cyst infection or remarkable abdominal pain. EUS-GTD was performed directly in the case with perforation due to cyst infection.

Since the rate of adverse events in our series is low (1.5\%), it can be said that EUS-GTD is a relatively safe procedure for pancreatic pseudocyst drainage. Although EUS-GTD is a safe and effective therapy method for drainage of pancreatic pseudocyst, prospective studies which compare EUS-GTD to surgery to evaluate the long-term outcome are required [9].

We had chosen external drainage mainly and in some cases, we chose internal drainage. Recently, we insert both internal and external drainage tube in one session of procedure. Several current studies recommended insertion of both internal and external drainage tube by using double guidewire technique $[10,11]$. There is no prospective study comparing efficacy between both internal and external tube placement at once or external tube or internal tube only. In our series, it was necessary to replace external drainage tube to internal drainage tube in 26 cases and to replace internal drainage tube to external drainage tube in 1 case. Since recannulating the pseudocyst is sometimes cumbersome, the combination placement of internal and external drainage tube using double guidewire technique will be an effective and reasonable method for pancreatic pseudocyst drainage.
Total recurrence rate in our study was $23.9 \%$. The current another study also reported that recurrence rate of fluid collection was $13.4 \%$ [12]. The reasons for recurrence may include not only stent condition but also any background of pancreas condition. We also analyzed several factors for recurrences; however, no particular factors were significant in our series. The result was not shown to be significant. The reason for this may be that this was a single center study, and was performed retrospectively. A larger prospective series study is required for detecting the factors for recurrence.

\section{Conclusion}

EUS-GTD is a relatively safe and effective therapeutic method. Therefore, EUS-GTD can be the first choice for therapy of pancreatic pseudocyst. However, further analysis should be done by a larger series to check the efficacy and safety and to determine the method of EUS-GTD for pancreatic pseudocyst.

\section{References}

[1] S. Varadarajulu, C. M. Wilcox, A. Tamhane, M. A. Eloubeidi, J. Blakely, and C. L. Canon, "Role of EUS in drainage of peripancreatic fluid collections not amenable for endoscopic transmural drainage," Gastrointestinal Endoscopy, vol. 66, no. 6, pp. 1107-1119, 2007.

[2] M. Krüger, A. S. Schneider, M. P. Manns, and P. N. Meier, "Endoscopic management of pancreatic pseudocysts or abscesses after an EUS-guided 1-step procedure for initial access," Gastrointestinal Endoscopy, vol. 63, no. 3, pp. 409-416, 2006.

[3] C. V. Lopes, C. Pesenti, E. Bories, F. Caillol, and M. Giovannini, "Endoscopic-ultrasound-guided endoscopic transmural drainage of pancreatic pseudocysts and abscesses," Scandinavian Journal of Gastroenterology, vol. 42, no. 4, pp. 524-529, 2007.

[4] M. R. Antillon, R. J. Shah, G. Stiegmann, and Y. K. Chen, "Single-step EUS-guided transmural drainage of simple and complicated pancreatic pseudocysts," Gastrointestinal Endoscopy, vol. 63, no. 6, pp. 797-803, 2006.

[5] S. Varadarajulu, "EUS followed by endoscopic pancreatic pseudocyst drainage or all-in-one procedure: a review of basic techniques (with video)," Gastrointestinal Endoscopy, vol. 69, no. 2, pp. S176-S181, 2009.

[6] R. Sadik, E. Kalaitzakis, A. Thune, J. Hansen, and C. Jönson, "Eus-guided drainage is more successful in pancreatic pseudocysts compared with abscesses," World Journal of Gastroenterology, vol. 17, no. 4, pp. 499-505, 2011.

[7] S. Varadarajulu, M. A. Phadnis, J. D. Christein, and C. M. Wilcox, "Multiple transluminal gateway technique for EUSguided drainage of symptomatic walled-off pancreatic necrosis," Gastrointestinal Endoscopy, vol. 74, no. 1, pp. 74-80, 2011.

[8] S. Varadarajulu, A. Tamhane, and J. Blakely, "Graded dilation technique for EUS-guided drainage of peripancreatic fluid collections: an assessment of outcomes and complications and technical proficiency (with video)," Gastrointestinal Endoscopy, vol. 68, no. 4, pp. 656-666, 2008.

[9] S. Seewald, T. L. Ang, M. Kida, K. Y. K. Teng, and N. Soehendra, "EUS 2008 Working Group document: evaluation of EUSguided drainage of pancreatic-fluid collections (with video)," 
Gastrointestinal Endoscopy, vol. 69, no. 2, supplement, pp. S13S21, 2009.

[10] T. Itoi, F. Itokawa, T. Tsuchiya, T. Kawai, and F. Moriyasu, "Eusguided pancreatic pseudocyst drainage: simultaneous placement of stents and nasocystic catheter using double-guidewire technique," Digestive Endoscopy, vol. 21, supplement s1, pp. S53S56, 2009.

[11] R. Puri, S. R. Mishra, R. B. Thandassery et al., "Outcome and complication of endoscopic ultrasound guided pancreatic pseudocyst drainage using combined endoprosthesis and nasocystic drain," Journal of Gastroenterology and Hepatology, vol. 27, no. 4, pp. 722-727, 2012.

[12] S. Seewald, T. L. Ang, H. Richter et al., "Long term results after endoscopic drainage and necrosectomy of symptomatic pancreatic fluid collection," Digestive Endoscopy, vol. 24, pp. 3641, 2011. 


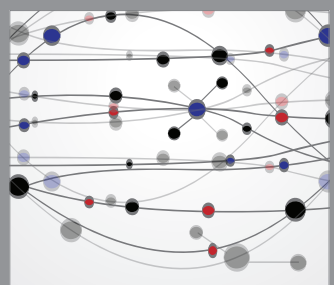

The Scientific World Journal
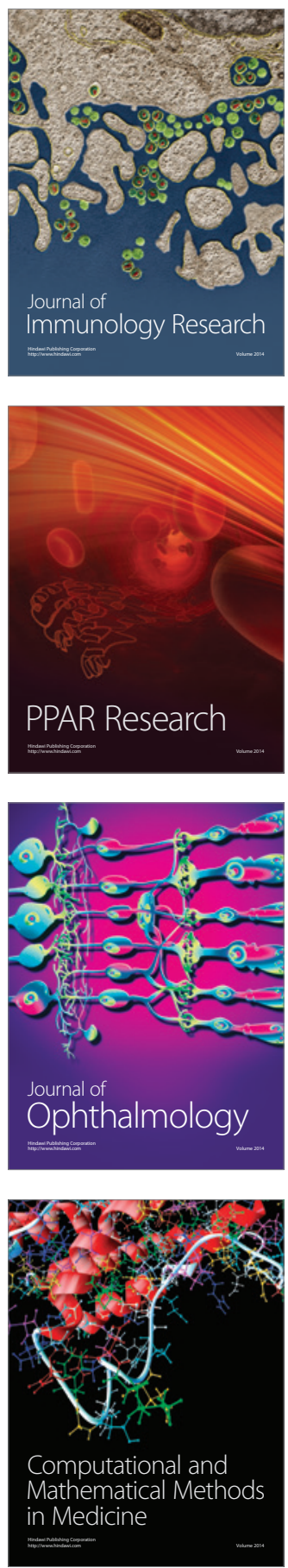

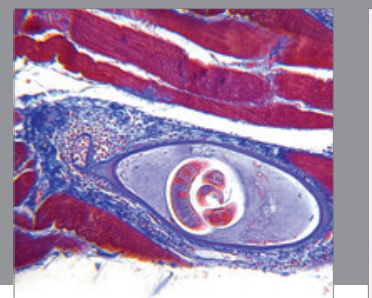

Gastroenterology

Research and Practice
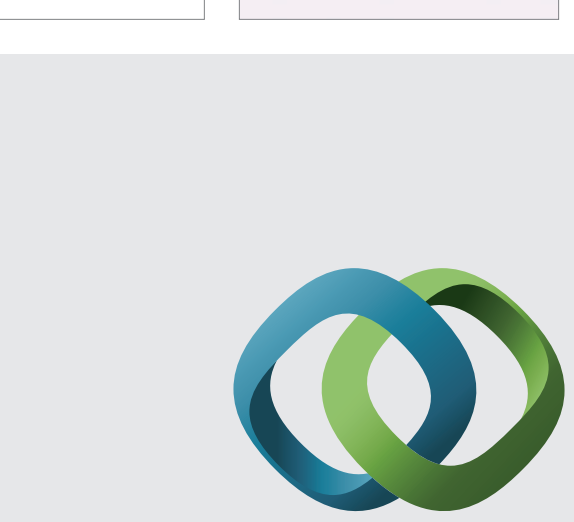

\section{Hindawi}

Submit your manuscripts at

http://www.hindawi.com
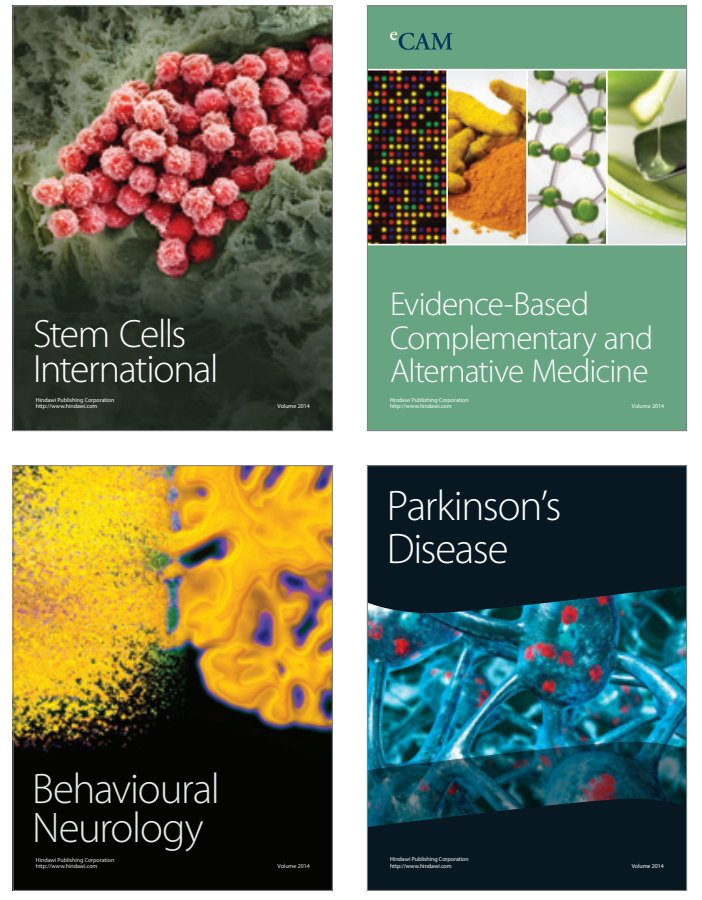
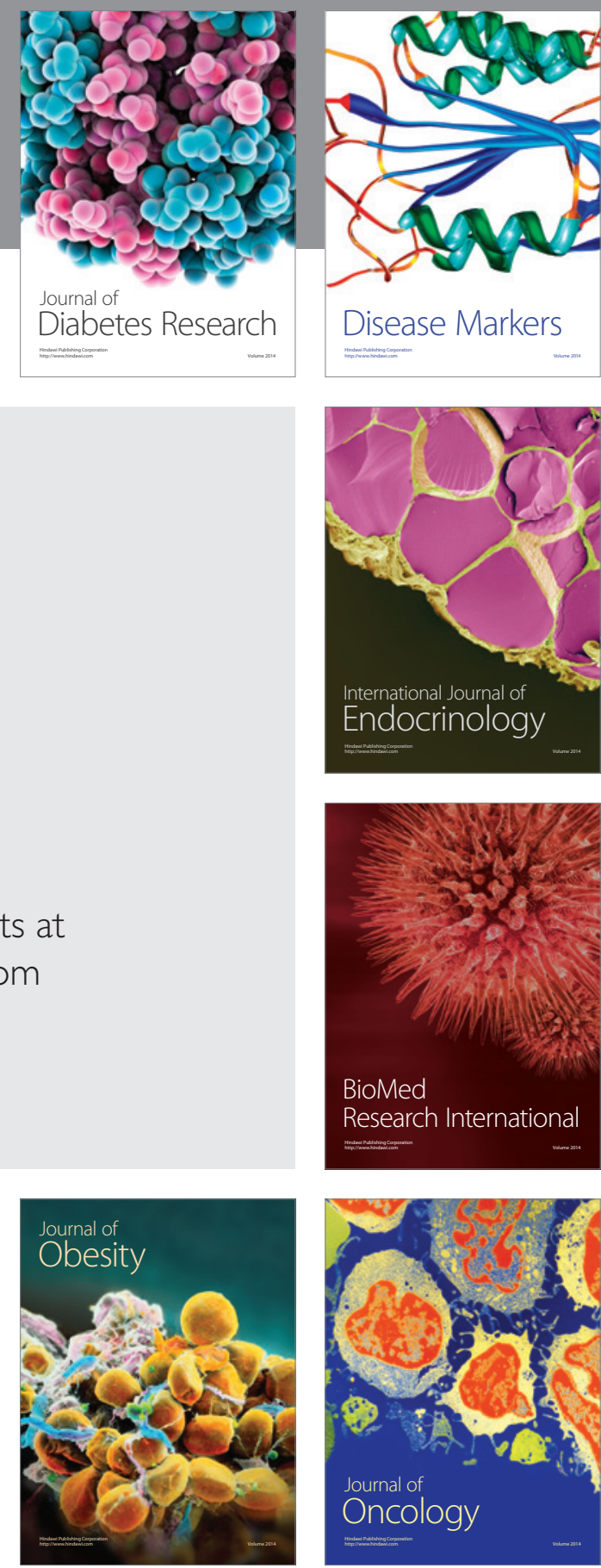

Disease Markers
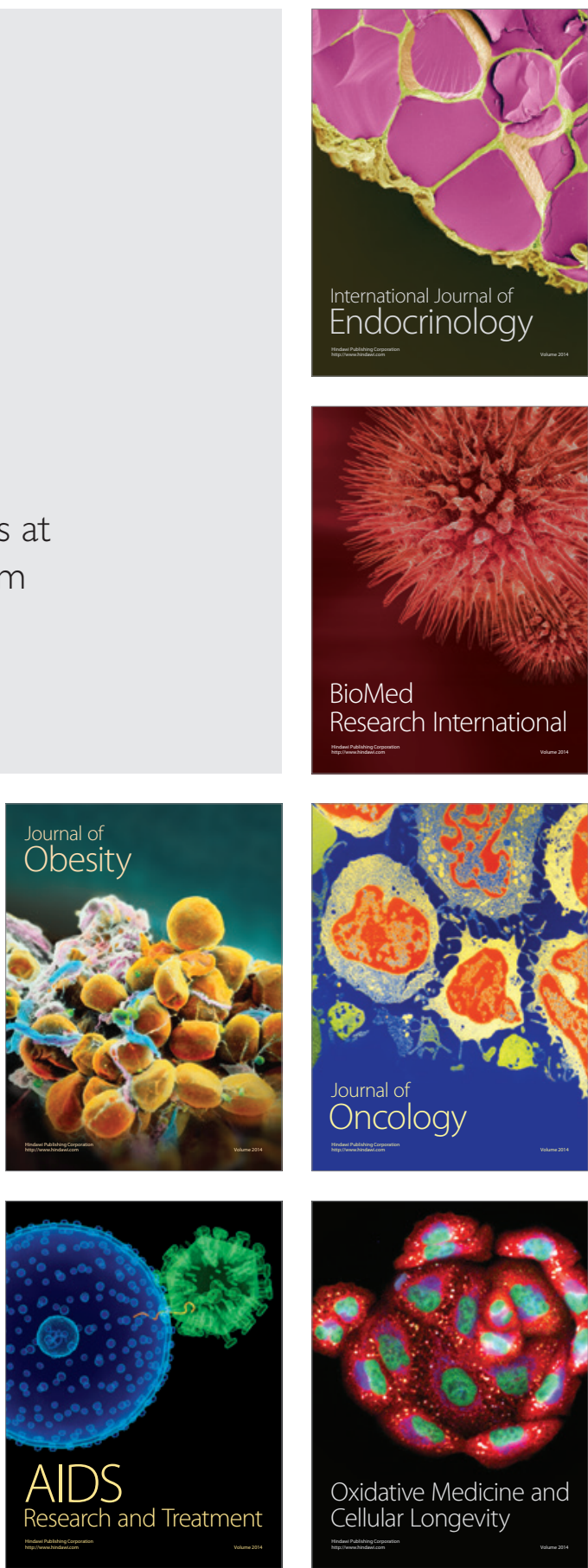